Economic analyses of breast cancer control in low- and middle-income countries: a systematic review

Zelle and Baltussen 


\title{
Economic analyses of breast cancer control in low- and middle-income countries: a systematic review
}

\author{
Sten G Zelle* and Rob M Baltussen
}

\begin{abstract}
Background: To support the development of global strategies against breast cancer, this study reviews available economic evidence on breast cancer control in low- and middle-income countries (LMICS).

Methods: A systematic article search was conducted through electronic scientific databases, and studies were included only if they concerned breast cancer, used original data, and originated from LMICs. Independent assessment of inclusion criteria yielded 24 studies that evaluated different kinds of screening, diagnostic, and therapeutic interventions in various age and risk groups. Studies were synthesized and appraised through the use of a checklist, designed for evaluating economic analyses.

Results: The majority of these studies were of poor quality, particularly in examining costs. Studies demonstrated the economic attractiveness of breast cancer screening strategies, and of novel treatment and diagnostic interventions.

Conclusions: This review shows that the evidence base to guide strategies for breast cancer control in LMICs is limited and of poor quality. The limited evidence base suggests that screening strategies may be economically attractive in LMICs - yet there is very little evidence to provide specific recommendations on screening by mammography versus clinical breast examination, the frequency of screening, or the target population. These results demonstrate the need for more economic analyses that are of better quality, cover a comprehensive set of interventions and result in clear policy recommendations.
\end{abstract}

Keywords: Breast cancer control, Economic evaluation, Systematic review, Low- and middle-income countries, Cost-effectiveness, Noncommunicable diseases

\section{Background}

Noncommunicable diseases (NCDs) have become increasingly important in low- and middle-income countries (LMICs). Once considered a problem only in high-income countries (HICs), more and more patients who suffer from cancers and other NCDs are now observed in LMICs [1]. This is mainly due to the ageing populations and changing lifestyles in LMICs [2]. The global importance of NCDs has recently been acknowledged through the UN Summit on NCDs, held by the UN General Assembly in September 2011. As highlighted in the summit, the most prominent

\footnotetext{
*Correspondence: S.Zelle@elg.umcn.nl

Department of Primary and Community Care, Radboud University Nijmegen Medical Centre, P.O. Box 9101 Internal Postal Code 117, 6500HB Nijmegen,
} the Netherlands cause of cancer death among women in LMICs is breast cancer, accounting for 269,000 deaths $(12.7 \%$ of all cancer deaths) in 2008 [3,4].

In HICs, many efforts have been undertaken to control breast cancer, leading to various improvements in breast cancer outcomes [5,6]. Strategies for breast cancer control are geared towards early detection and early treatment, and although its benefits are still open to discussion [7-9], mammography screening has been widely implemented [10-12]. In these countries, the selection of breast cancer control strategies has often been guided by economic analyses, demonstrating the value of alternative interventions [13-16].

In contrast to the established breast cancer control strategies in HICs, breast cancer is often neglected in

\section{Biomed Central}


LMICs and control strategies lack evidence-based information [17-20]. Policy-makers in LMICs cannot adopt similar breast cancer control strategies as implemented in HICs because most LMICs rely on much smaller budgets, and both the costs and effectiveness of control strategies are highly dependent on the population characteristics and the functioning of the health system $[11,20,21]$.

Against this background, the present review provides an inventory of economic analyses of breast cancer control in LMICs. The paper's objectives are to present the available economic evidence from LMICs and to assess the methodological quality of the analyses. This research could improve the evidence base on cost-effective breast cancer interventions and could strengthen breast cancer control policy in LMICs.

\section{Methods}

\section{Search strategy and selection criteria}

In this review, we analyzed publications from the MEDLINE index using PubMed, the Web of Science, Scopus, and Google Scholar. We searched the literature using the keyword 'breast cancer', combined with the keywords: 'developing countries', 'Asia, 'USSR', 'MiddleEast,' 'Eastern Europe,' 'West-Indies,' 'China,' 'Russia,' 'India,' 'Africa, or 'limited resource', or combined with: 'cost-benefit', 'cost-effectiveness', 'costing', or 'cost analysis'. Additionally, we searched these indexes using 'breast neoplasms', 'developing countries', and 'economics' in MeSH terms. Our search took place in January 2013, and was limited to publications in English. Studies were included only if they concerned breast cancer and originated from LMICs as listed by The World Bank [22].

The selection process is shown in Figure 1. In step 1, articles found by our search in the various indexes were merged in a database, which was then corrected for duplications (in Google Scholar, because of the large number of articles founds, we screened titles until the point that we did not find any further relevant title among the last 500 screened titles; in total, we screened 800 titles in this database). In step 2 we screened the titles of these articles, in step 3 the abstracts and in step 4 the remaining articles were read completely. We excluded publications for which no full-text article versions were available, or those not published in English. Furthermore, we excluded articles that only mentioned costs or cost-effectiveness without presenting original data.

\section{Study characteristics}

We documented the following characteristics from the reviewed articles: country or region, base year of cost data, study population, and breast cancer stage(s) considered. The stage was categorized as stage I to IV according to the American Joint Committee on Cancer [23].
We documented the following methodological characteristics: type of economic evaluation -cost analysis or cost of illness analysis, separately reported costs and effects, cost-effectiveness analysis, cost-benefit analysis, and cost-utility analysis; study design - experimental, observational (cohort, case control, or cross-sectional), model based, and other designs; study perspective non-healthcare perspective (for example, productivity loss, travel costs, co-payments), healthcare perspective (for example, hospital administration costs, treatment costs), and societal perspective including non-healthcare and healthcare costs; time horizon; and outcome measure for effectiveness (disability-adjusted life years, qualityadjusted life years, life years saved, lives saved, and intermediate outcome measures).

The following qualitative characteristics were documented: sources for estimation of effectiveness, sources for estimation of resource utilization, discount rates used, sensitivity analysis for assumptions, and reported incremental analysis. We classified sources for estimation of effectiveness and resource utilization by primary data collection (for example, patients, questionnaires), secondary data collection (for example, records), literature based, expert opinion, and other. We also noted whether discount rates were used on costs, effects, both costs and effects, or not at all.

We also registered the study objective, the evaluated interventions, and the main study conclusions for each reviewed article.

\section{Study evaluation}

We used an established checklist by Drummond and Jefferson to judge the quality of the economic evaluations $[24,25]$. A three-point response scale was added, similar to Gerard and colleagues [25], to more specifically grade the quality of each item on the checklist. Scores on this scale ranged from 0 (not considered), to 1 (partially considered) to 2 (fully considered). A few adjustments to the checklist by Drummond and Jefferson were necessary to create a more responsive scoring system for our particular set of economic studies. We removed those items that were not applicable to any of the reviewed studies (for example, on productivity changes), and combined some items that were otherwise putting too much emphasis to certain domains in the overall score (for example, on health state valuations and discount rates). The adapted checklist is provided in Table 1. We summed up all scores, and compared this with the maximum attainable score to calculate the mean quality score of a study (as a percentage of the maximum attainable score). We accounted for items that were not relevant to the study under scrutiny (for example, studies that studied costs and effects in a single year were not criticized for not applying any discount rate in the analyses). 


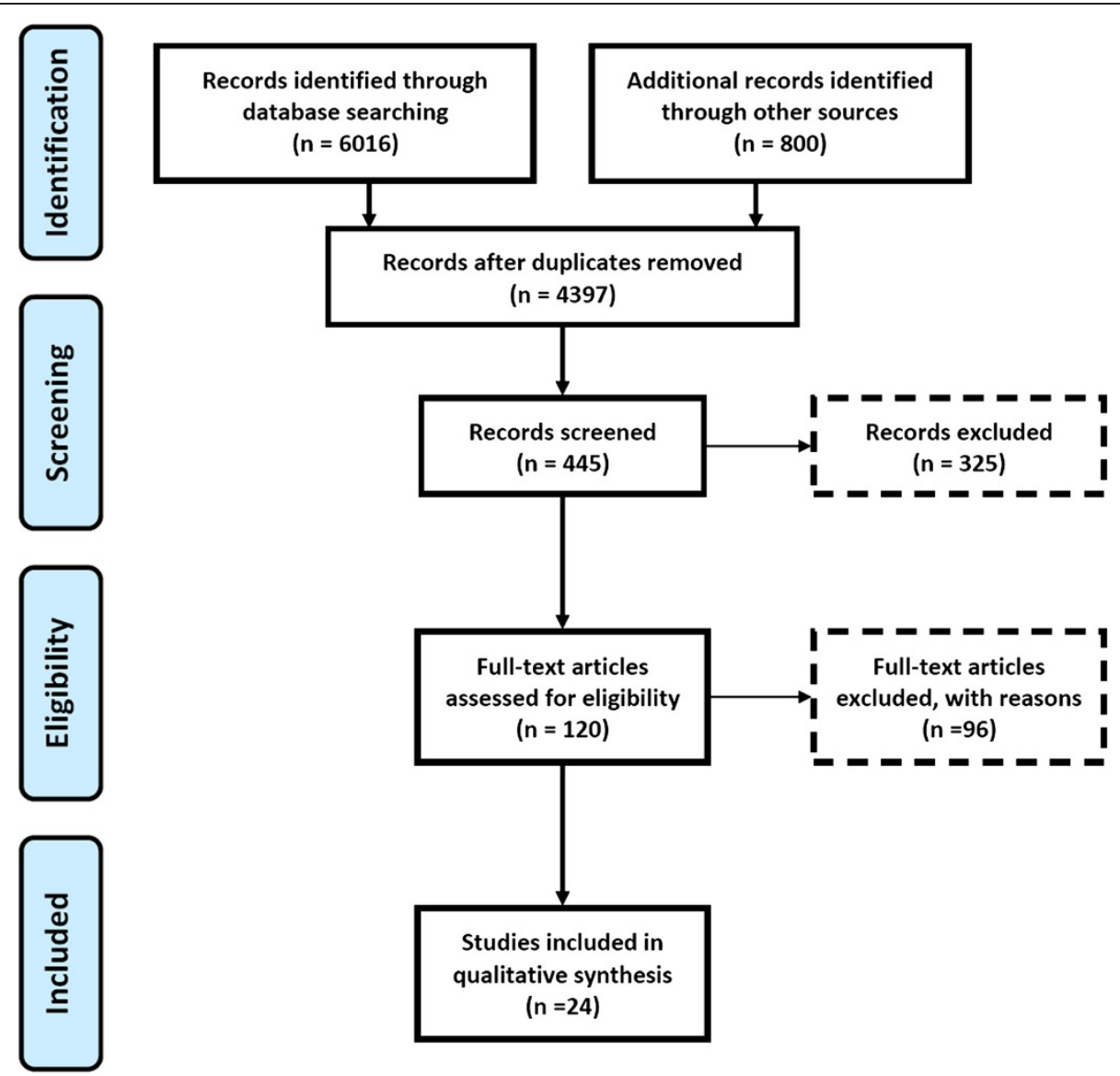

Figure 1 Prisma statement 1: Prisma 2009 flow diagram.

Two reviewers (SGZ and RMB) evaluated each publication for conformance with this checklist, and consensus was reached when scores differed. We followed PRISMA guidelines for reporting this systematic review.

\section{Results}

Search results

The stepwise selection of articles by our selection criteria is presented in Figure 1. Our search strategy resulted in a total of 6,816 studies: 679 studies from PubMed, 328 studies from Web of Science, 5,009 studies from Scopus, and 800 from Google Scholar, respectively. In step 1, by merging the results of all individual search strategies and excluding duplication, the total number of hits was reduced to almost 4,400 . Upon screening of titles (step 2), abstracts (step 3) and full texts (step 4), we eventually identified 24 articles that met our inclusion criteria.

\section{Study characteristics}

Table 2 describes the baseline characteristics of the 24 included studies. We found eight studies from Asia, most concerning China, India and Iran. Five studies were on a global or sub-regional level, while there were five studies from Africa, three from Europe and three from Latin America. A total of 10 studies evaluated breast cancer screening in combination with treatment ( $n=10)$, assessing mammography screening $(n=9)$, clinical breast examination (CBE) $(n=3)$, magnetic resonance imaging $(n=1)$, ultrasound $(n=1)$, biopsy $(n=1)$, elasticity imaging $(n=1)$, and tactile imaging $(n=1)$, respectively [26-36]. These studies evaluated a variety of age groups and screening frequencies (Table 3). One study reported on a mass-media intervention to improve the early detection of breast cancer in Ghana [35]. Seven studies evaluated only treatment interventions including drug therapy $(n=4)$, oophorectomy $(n=1)$, radiotherapy $(n=1)$, and treatment in general $(n=1)$ [37-42]. Other studies examined the costs of diagnostic interventions $(n=3)$ or did not consider a specific intervention $(n=2)$ [43-48].

The methodological study characteristics of the reviewed studies are presented in Table 2. The base year of cost data in the included studies was generally not from before year 2000, and could not be identified in eight studies. The majority of studies combined both costs and effects in a 
Table 1 Checklist for quality of economic evaluations

\begin{tabular}{|c|c|c|c|c|}
\hline Item & Fully & Partial & Not at all & Not appropriate \\
\hline Original checklist & 2 points & 1 point & 0 points & NA \\
\hline \multicolumn{5}{|l|}{ Study design } \\
\hline 1. The research question is stated & $\square$ & $\square$ & $\square$ & $\square$ \\
\hline 2. The economic importance of the research question is stated & $\square$ & $\square$ & $\square$ & $\square$ \\
\hline $\begin{array}{l}\text { 3. The viewpoint(s) of the analysis are clearly stated and justified } \\
\text { (relating to a particular decision-making context) }\end{array}$ & $\square$ & $\square$ & $\square$ & $\square$ \\
\hline $\begin{array}{l}\text { 4. The rationale(s) for choosing the alternative programs or interventions which } \\
\text { are compared is stated }\end{array}$ & $\square$ & $\square$ & $\square$ & $\square$ \\
\hline 5. The alternatives being compared are clearly described & $\square$ & $\square$ & $\square$ & $\square$ \\
\hline 6. All relevant alternatives are included & $\square$ & $\square$ & $\square$ & $\square$ \\
\hline 7. The choice of economic evaluation is justified in relation to the questions addressed & $\square$ & $\square$ & $\square$ & $\square$ \\
\hline \multicolumn{5}{|l|}{ Effectiveness estimation } \\
\hline 8. The primary outcome measure for the economic evaluation is clearly stated & $\square$ & $\square$ & $\square$ & $\square$ \\
\hline 9. The source(s) of effectiveness estimates used is clearly stated & $\square$ & $\square$ & $\square$ & $\square$ \\
\hline $\begin{array}{l}\text { 10. Details of the design and results of the effectiveness study are given } \\
\text { (if based on a single study) }\end{array}$ & $\square$ & $\square$ & $\square$ & $\square$ \\
\hline $\begin{array}{l}\text { 11. Details of the methods of synthesis or meta-analysis of estimates are given } \\
\text { (if based on multiple studies) }\end{array}$ & $\square$ & $\square$ & $\square$ & $\square$ \\
\hline 12. Data and methods used to value health states and other benefits are stated and justified. & $\square$ & $\square$ & $\square$ & $\square$ \\
\hline \multicolumn{5}{|l|}{ Cost estimation } \\
\hline 14. Indirect non-healthcare costs are included or discussed & $\square$ & $\square$ & $\square$ & $\square$ \\
\hline 15. Quantities of resources are reported separately from their unit costs & $\square$ & $\square$ & $\square$ & $\square$ \\
\hline 16. Methods for the estimation of quantities and unit costs are described and justified. & $\square$ & $\square$ & $\square$ & $\square$ \\
\hline 17. Details of currency of price adjustments for inflation or currency conversion are given & $\square$ & $\square$ & $\square$ & $\square$ \\
\hline \multicolumn{5}{|l|}{ Analysis } \\
\hline 18. Time horizon of costs and benefits are stated & $\square$ & $\square$ & $\square$ & $\square$ \\
\hline 18. Details of any model used are given & $\square$ & $\square$ & $\square$ & $\square$ \\
\hline 19. The choice of model used and the key parameters on which it is based are justified & $\square$ & $\square$ & $\square$ & $\square$ \\
\hline 20. The discount rate(s) is stated & $\square$ & $\square$ & $\square$ & $\square$ \\
\hline 21. The choice of rate(s) is justified & $\square$ & $\square$ & $\square$ & $\square$ \\
\hline 22. Details of statistical tests and confidence intervals are given for stochastic data & $\square$ & $\square$ & $\square$ & $\square$ \\
\hline $\begin{array}{l}\text { 23. Sensitivity analysis is performed: } \\
\text { 1) Deterministic (one way /multiple way) }\end{array}$ & $\square$ & $\square$ & $\square$ & $\square$ \\
\hline $\begin{array}{l}\text { 24. The choice of variables in sensitivity analysis and the range over which these variables } \\
\text { are varied is justified }\end{array}$ & $\square$ & $\square$ & $\square$ & $\square$ \\
\hline 25. Incremental analysis is performed and reported & $\square$ & $\square$ & $\square$ & $\square$ \\
\hline Interpretation of results & $\square$ & $\square$ & $\square$ & $\square$ \\
\hline 26. Major outcomes are presented in a disaggregated as well as aggregated form & $\square$ & $\square$ & $\square$ & $\square$ \\
\hline 27. The answer to the study question is given & $\square$ & $\square$ & $\square$ & $\square$ \\
\hline 28. Relevant alternatives are compared & $\square$ & $\square$ & $\square$ & $\square$ \\
\hline 29. Conclusions follow from the data reported & $\square$ & $\square$ & $\square$ & $\square$ \\
\hline $\begin{array}{l}\text { 30. Conclusions are accompanied by the appropriate caveats such as generalizability, } \\
\text { equity, feasibility, and implementation }\end{array}$ & $\square$ & $\square$ & $\square$ & $\square$ \\
\hline
\end{tabular}

This checklist was adapted from Drummond and Jefferson [24].

single cost-effectiveness estimate $(n=13)$, and the majority of these were based on mathematical models $(n=9)$. Most studies used a healthcare perspective $(n=19)$, and only one study included non-healthcare costs [48]. Studies used a time horizon varying between 5 weeks and the lifetime of the study population. Most reviewed studies used intermediate outcome measures (that is, clinical effects $n=8$ ), life years saved $(n=6)$, or disability-adjusted life years $(n=5)$ as their main effectiveness outcome, while qualityadjusted life years were less frequently used $(n=3)$. 
Table 2 Characteristics of reviewed studies, ordered by base year of cost data

\begin{tabular}{|c|c|c|c|c|c|c|c|}
\hline Authors & $\begin{array}{l}\text { Region / } \\
\text { country }\end{array}$ & $\begin{array}{l}\text { Base year } \\
\text { of cost data }\end{array}$ & Study population & $\begin{array}{l}\text { Breast } \\
\text { cancer } \\
\text { stage } \\
\text { considered }\end{array}$ & $\begin{array}{l}\text { Economic } \\
\text { evaluation } \\
\text { type }\end{array}$ & Study design & Perspective \\
\hline $\begin{array}{l}\text { Groot and } \\
\text { colleagues, } 2006[28]\end{array}$ & $\begin{array}{l}\text { World } \\
\text { sub-regions }\end{array}$ & 2000 & $\begin{array}{l}\text { Female population at risk, } \\
\text { in AfrE, AmroA, SearD }\end{array}$ & All & $\begin{array}{l}\text { Cost-effectiveness } \\
\text { analysis }\end{array}$ & Model based & Healthcare \\
\hline $\begin{array}{l}\text { Okonkwo and } \\
\text { colleagues, } 2008 \text { [30] }\end{array}$ & India & 2001 & Female population at risk & All & $\begin{array}{l}\text { Cost-effectiveness } \\
\text { analysis }\end{array}$ & Model based & Healthcare \\
\hline Munshi, 2009 [41] & Worldwide & $\begin{array}{l}\text { Varying from } \\
2002 \text { to } 2007\end{array}$ & $\begin{array}{l}\text { Breast cancer patients } \\
\text { in general }\end{array}$ & All & $\begin{array}{l}\text { Report on costs and } \\
\text { effects separately }\end{array}$ & Other & Healthcare \\
\hline $\begin{array}{l}\text { Sarvazyan and } \\
\text { colleagues, } 2008 \text { [32] }\end{array}$ & Worldwide & $\begin{array}{l}\text { Varying from } \\
2003 \text { to } 2007\end{array}$ & Female population at risk & All & $\begin{array}{l}\text { Cost-effectiveness } \\
\text { analysis }\end{array}$ & Other & Not stated \\
\hline $\begin{array}{l}\text { Fonseca and } \\
\text { colleagues, } 2009 \text { [38] }\end{array}$ & Brazil & 2005 & $\begin{array}{l}\text { Hypothetical cohort of } 64- \\
\text { year-old postmenopausal } \\
\text { women }\end{array}$ & All & $\begin{array}{l}\text { Cost-effectiveness } \\
\text { analysis }\end{array}$ & Model based & Healthcare \\
\hline $\begin{array}{l}\text { Ginsberg and } \\
\text { colleagues, } 2012 \text { [27] }\end{array}$ & $\begin{array}{l}\text { Sub-Saharan } \\
\text { Africa and } \\
\text { South East Asia }\end{array}$ & 2005 & $\begin{array}{l}\text { Female population at risk, } \\
\text { in SearD and AfrE }\end{array}$ & All & $\begin{array}{l}\text { Cost-effectiveness } \\
\text { analysis }\end{array}$ & Model based & Healthcare \\
\hline $\begin{array}{l}\text { Salomon and } \\
\text { colleagues, } 2012 \text { [31] }\end{array}$ & Mexico & 2005 & Female population at risk & All & $\begin{array}{l}\text { Cost-effectiveness } \\
\text { analysis }\end{array}$ & Model based & Healthcare \\
\hline $\begin{array}{l}\text { Pakseresht and } \\
\text { colleagues, } 2011 \text { [48] }\end{array}$ & India & $2006 / 2007$ & $\begin{array}{l}103 \text { women with primary } \\
\text { breast cancer in a tertiary } \\
\text { hospital }\end{array}$ & All & $\begin{array}{l}\text { Cost analysis/cost } \\
\text { of illness }\end{array}$ & Observational & $\begin{array}{l}\text { Non- } \\
\text { healthcare }\end{array}$ \\
\hline $\begin{array}{l}\text { Yazihan and } \\
\text { Yilmaz, } 2006 \text { [34] }\end{array}$ & Turkey & 2007 & Female population at risk & All & $\begin{array}{l}\text { Cost-effectiveness } \\
\text { analysis }\end{array}$ & Other & Healthcare \\
\hline $\begin{array}{l}\text { Bastani and } \\
\text { Kiadaliri, } 2012 \text { [49] }\end{array}$ & Iran & 2008 & $\begin{array}{l}\text { Patients younger than } 75 \\
\text { with node-positive breast } \\
\text { cancer }\end{array}$ & All & Cost-utility analysis & Experimental & Healthcare \\
\hline $\begin{array}{l}\text { Liubao and } \\
\text { colleagues, } 2009 \text { [39] }\end{array}$ & China & 2008 & $\begin{array}{l}\text { Model cohort of 1,000 } \\
51 \text {-year-old operable } \\
\text { breast cancer patients }\end{array}$ & All & $\begin{array}{l}\text { Cost-effectiveness } \\
\text { analysis }\end{array}$ & Model based & Healthcare \\
\hline Astim, 2011 [36] & Turkey & 2010 & $\begin{array}{l}\text { Female population at } \\
\text { risk older than } 30\end{array}$ & All & $\begin{array}{l}\text { Report on costs and } \\
\text { effects separately }\end{array}$ & Model based & Healthcare \\
\hline $\begin{array}{l}\text { Zelle and colleagues, } \\
2012 \text { [35] }\end{array}$ & Ghana & 2010 & Female population at risk & All & $\begin{array}{l}\text { Cost-effectiveness } \\
\text { analysis }\end{array}$ & Model based & Healthcare \\
\hline $\begin{array}{l}\text { Bai and colleagues, } \\
2012 \text { [42] }\end{array}$ & China & 2012 & $\begin{array}{l}\text { Model cohort of women aged } \\
51.7 \text {, with early stage breast } \\
\text { cancer after lumpectomy }\end{array}$ & 1 and 2 & $\begin{array}{l}\text { Cost-effectiveness } \\
\text { analysis }\end{array}$ & Model based & Healthcare \\
\hline $\begin{array}{l}\text { Arredondo and } \\
\text { colleagues, } 1995 \text { [43] }\end{array}$ & Brazil & Not clear & $\begin{array}{l}\text { Hypothetical breast } \\
\text { cancer case }\end{array}$ & All & $\begin{array}{l}\text { Cost analysis/cost } \\
\text { of illness }\end{array}$ & Observational & Healthcare \\
\hline $\begin{array}{l}\text { Boutayeb and } \\
\text { colleagues, } 2010 \text { [37] }\end{array}$ & Morocco & Not clear & $\begin{array}{l}\text { Early-stage breast cancer } \\
\text { patients in Morocco }\end{array}$ & Not clear & $\begin{array}{l}\text { Cost-effectiveness } \\
\text { analysis }\end{array}$ & Observational & Healthcare \\
\hline $\begin{array}{l}\text { Denewer and } \\
\text { colleagues, } 2010 \text { [26] }\end{array}$ & Egypt & Not clear & $\begin{array}{l}\text { Female population at risk } \\
\text { between } 25 \text { and } 65 \text { years }\end{array}$ & All & $\begin{array}{l}\text { Report on costs and } \\
\text { effects separately }\end{array}$ & Experimental & Healthcare \\
\hline $\begin{array}{l}\text { Guggisberg and } \\
\text { colleagues, } 2011 \text { [46] }\end{array}$ & Cameroon & Not clear & $\begin{array}{l}\text { Women who underwent } \\
\text { FNA in a rural hospital }\end{array}$ & All & $\begin{array}{l}\text { Report on costs and } \\
\text { effects separately }\end{array}$ & Observational & Healthcare \\
\hline Kobayashi, 1988 [44] & Worldwide & Not clear & NA & $\mathrm{NA}$ & $\begin{array}{l}\text { Cost analysis/cost } \\
\text { of illness }\end{array}$ & Observational & Healthcare \\
\hline $\begin{array}{l}\text { Love and colleagues, } \\
2002 \text { [40] }\end{array}$ & $\begin{array}{l}\text { Vietnam } \\
\text { and China }\end{array}$ & Not clear & $\begin{array}{l}\text { Premenopausal Vietnamese } \\
\text { and Chinese breast cancer } \\
\text { patients, considered for surgery }\end{array}$ & 2 & $\begin{array}{l}\text { Cost-effectiveness } \\
\text { analysis }\end{array}$ & Experimental & Healthcare \\
\hline $\begin{array}{l}\text { Mousavi and } \\
\text { colleagues, } 2008 \text { [29] }\end{array}$ & Iran & Not clear & $\begin{array}{l}\text { Female population at } \\
\text { risk between } 35 \text { and } 69\end{array}$ & All & $\begin{array}{l}\text { Report on costs and } \\
\text { effects separately }\end{array}$ & Other & Healthcare \\
\hline $\begin{array}{l}\text { Nasrinossadat and } \\
\text { colleagues, } 2011 \text { [47] }\end{array}$ & Iran & Not clear & $\begin{array}{l}51 \text { patients that underwent } \\
\text { surgical excision of } \\
\text { nonpalpable breast masses }\end{array}$ & All & $\begin{array}{l}\text { Report on costs and } \\
\text { effects separately }\end{array}$ & Observational & Healthcare \\
\hline $\begin{array}{l}\text { Thomas and } \\
\text { colleagues, } 1999 \text { [45] }\end{array}$ & Nigeria & Not clear & $\begin{array}{l}\text { Patients who received FNA } \\
\text { between } 1994 \text { and } 1997\end{array}$ & All & $\begin{array}{l}\text { Report on costs and } \\
\text { effects separately }\end{array}$ & Observational & Patient \\
\hline
\end{tabular}


Table 2 Characteristics of reviewed studies, ordered by base year of cost data (Continued)

\begin{tabular}{|c|c|c|c|c|c|c|c|c|}
\hline Authors & Perspective & $\begin{array}{l}\text { Time } \\
\text { horizon }\end{array}$ & $\begin{array}{l}\text { Effectiveness } \\
\text { outcome } \\
\text { measure }\end{array}$ & $\begin{array}{l}\text { Sources for } \\
\text { estimation of } \\
\text { effectiveness }\end{array}$ & $\begin{array}{l}\text { Sources for } \\
\text { estimation } \\
\text { of resource } \\
\text { utilization }\end{array}$ & $\begin{array}{l}\text { Discount } \\
\text { rates used }\end{array}$ & $\begin{array}{l}\text { Sensitivity } \\
\text { analysis for } \\
\text { assumptions } \\
\text { presented }\end{array}$ & $\begin{array}{l}\text { Incremental } \\
\text { analysis } \\
\text { reported }\end{array}$ \\
\hline $\begin{array}{l}\text { Groot and } \\
\text { colleagues, } 2006 \text { [28] }\end{array}$ & Healthcare & $\begin{array}{l}100 \\
\text { years }\end{array}$ & DALYS & Literature based & $\begin{array}{l}\text { Secondary } \\
\text { data collection }\end{array}$ & $\begin{array}{l}\text { On both costs } \\
\text { and effects }\end{array}$ & Yes & Yes \\
\hline $\begin{array}{l}\text { Okonkwo and } \\
\text { colleagues, } 2008 \text { [30] }\end{array}$ & Healthcare & 25 years & Life years saved & $\begin{array}{l}\text { Secondary data } \\
\text { collection }\end{array}$ & $\begin{array}{l}\text { Secondary } \\
\text { data collection }\end{array}$ & $\begin{array}{l}\text { On both costs } \\
\text { and effects }\end{array}$ & Yes & Yes \\
\hline Munshi, 2009 [41] & Healthcare & NA & $\begin{array}{l}\text { Intermediate } \\
\text { outcome } \\
\text { measures }\end{array}$ & Literature based & Literature & NA & NA & $\mathrm{NA}$ \\
\hline $\begin{array}{l}\text { Sarvazyan and } \\
\text { colleagues, } 2008 \text { [32] }\end{array}$ & Not stated & 1 year & Life years saved & Literature based & Literature & NA & Yes & No \\
\hline $\begin{array}{l}\text { Fonseca and } \\
\text { colleagues, } 2009 \text { [38] }\end{array}$ & Healthcare & Lifetime & Life years saved & Literature based & Expert opinion & $\begin{array}{l}\text { On both costs } \\
\text { and effects }\end{array}$ & Yes & Yes \\
\hline $\begin{array}{l}\text { Ginsberg and } \\
\text { colleagues, } 2012 \text { [27] }\end{array}$ & Healthcare & $\begin{array}{l}100 \\
\text { years }\end{array}$ & DALYS & Literature based & $\begin{array}{l}\text { Secondary } \\
\text { data collection }\end{array}$ & $\begin{array}{l}\text { On both costs } \\
\text { and effects }\end{array}$ & Yes & Yes \\
\hline $\begin{array}{l}\text { Salomon and } \\
\text { colleagues, } 2012 \text { [31] }\end{array}$ & Healthcare & $\begin{array}{l}100 \\
\text { years }\end{array}$ & DALYS & Literature based & $\begin{array}{l}\text { Secondary } \\
\text { data collection }\end{array}$ & $\begin{array}{l}\text { On both costs } \\
\text { and effects }\end{array}$ & Yes & Yes \\
\hline $\begin{array}{l}\text { Pakseresht and } \\
\text { colleagues, } 2011 \text { [48] }\end{array}$ & $\begin{array}{l}\text { Non- } \\
\text { healthcare }\end{array}$ & 2 years & NA & NA & $\begin{array}{l}\text { Primary data } \\
\text { collection }\end{array}$ & NA & NA & NA \\
\hline $\begin{array}{l}\text { Yazihan and } \\
\text { Yilmaz, } 2006 \text { [34] }\end{array}$ & Healthcare & 6 years & DALYS & $\begin{array}{l}\text { Secondary data } \\
\text { collection }\end{array}$ & $\begin{array}{l}\text { Secondary } \\
\text { data collection }\end{array}$ & None & No & No \\
\hline $\begin{array}{l}\text { Bastani and } \\
\text { Kiadaliri, } 2012 \text { [49] }\end{array}$ & Healthcare & $\begin{array}{l}8 \\
\text { months }\end{array}$ & QALYS & $\begin{array}{l}\text { Primary data } \\
\text { collection }\end{array}$ & $\begin{array}{l}\text { Primary data } \\
\text { collection }\end{array}$ & NA & No & NA \\
\hline $\begin{array}{l}\text { Liubao and } \\
\text { colleagues, } 2009 \text { [39] }\end{array}$ & Healthcare & Lifetime & QALYS & $\begin{array}{l}\text { Secondary data } \\
\text { collection }\end{array}$ & $\begin{array}{l}\text { Secondary } \\
\text { data collection }\end{array}$ & $\begin{array}{l}\text { On both costs } \\
\text { and effects }\end{array}$ & Yes & Yes \\
\hline Astim, 2011 [36] & Healthcare & 10 years & $\begin{array}{l}\text { Intermediate } \\
\text { outcome } \\
\text { measures }\end{array}$ & $\begin{array}{l}\text { Secondary data } \\
\text { collection }\end{array}$ & Literature & Yes & No & No \\
\hline $\begin{array}{l}\text { Zelle and colleagues, } \\
2012 \text { [35] }\end{array}$ & Healthcare & $\begin{array}{l}100 \\
\text { years }\end{array}$ & DALYS & Literature based & $\begin{array}{l}\text { Primary data } \\
\text { collection }\end{array}$ & $\begin{array}{l}\text { On both costs } \\
\text { and effects }\end{array}$ & Yes & Yes \\
\hline $\begin{array}{l}\text { Bai and colleagues, } \\
2012 \text { [42] }\end{array}$ & Healthcare & Lifetime & QALYS & Literature based & $\begin{array}{l}\text { Literature/ } \\
\text { expert opinion }\end{array}$ & $\begin{array}{l}\text { On both costs } \\
\text { and effects }\end{array}$ & Yes & Yes \\
\hline $\begin{array}{l}\text { Arredondo and } \\
\text { colleagues, } 1995 \text { [43] }\end{array}$ & Healthcare & NA & NA & NA & Expert opinion & NA & No & No \\
\hline $\begin{array}{l}\text { Boutayeb and } \\
\text { colleagues, } 2010 \text { [37] }\end{array}$ & Healthcare & 1 year & Life years saved & Literature based & $\begin{array}{l}\text { Secondary } \\
\text { data collection }\end{array}$ & NA & No & No \\
\hline $\begin{array}{l}\text { Denewer and } \\
\text { colleagues, } 2010 \text { [26] }\end{array}$ & Healthcare & 2 years & $\begin{array}{l}\text { Intermediate } \\
\text { outcome } \\
\text { measures }\end{array}$ & $\begin{array}{l}\text { Primary data } \\
\text { collection }\end{array}$ & Not clear & None & No & No \\
\hline $\begin{array}{l}\text { Guggisberg and } \\
\text { colleagues, } 2011 \text { [46] }\end{array}$ & Healthcare & 5 weeks & $\begin{array}{l}\text { Intermediate } \\
\text { outcome } \\
\text { measures }\end{array}$ & $\begin{array}{l}\text { Primary data } \\
\text { collection }\end{array}$ & Not clear & NA & No & No \\
\hline Kobayashi, 1988 [44] & Healthcare & NA & $\begin{array}{l}\text { Intermediate } \\
\text { outcome } \\
\text { measures }\end{array}$ & $\begin{array}{l}\text { Primary data } \\
\text { collection }\end{array}$ & $\begin{array}{l}\text { Primary data } \\
\text { collection }\end{array}$ & NA & NA & NA \\
\hline $\begin{array}{l}\text { Love and colleagues, } \\
2002[40]\end{array}$ & Healthcare & 15 years & Life years saved & $\begin{array}{l}\text { Primary data } \\
\text { collection }\end{array}$ & Not clear & $\begin{array}{l}\text { On both costs } \\
\text { and effects }\end{array}$ & No & Yes \\
\hline $\begin{array}{l}\text { Mousavi and } \\
\text { colleagues, } 2008 \text { [29] }\end{array}$ & Healthcare & 1 year & Life years saved & Expert opinion & Expert opinion & NA & No & No \\
\hline $\begin{array}{l}\text { Nasrinossadat and } \\
\text { colleagues, } 2011 \text { [47] }\end{array}$ & Healthcare & $\begin{array}{l}3 \text { to } 4 \\
\text { years }\end{array}$ & $\begin{array}{l}\text { Intermediate } \\
\text { outcome } \\
\text { measures }\end{array}$ & $\begin{array}{l}\text { Primary data } \\
\text { collection }\end{array}$ & Not clear & None & No & No \\
\hline $\begin{array}{l}\text { Thomas and } \\
\text { colleagues, } 1999 \text { [45] }\end{array}$ & Patient & NA & $\begin{array}{l}\text { Intermediate } \\
\text { outcome measures }\end{array}$ & $\begin{array}{l}\text { Primary data } \\
\text { collection }\end{array}$ & Not clear & NA & NA & NA \\
\hline
\end{tabular}


Table 3 Interventions compared, study objectives, and main study conclusions of reviewed articles

\begin{tabular}{lll}
\hline Authors & Interventions compared & Study objective \\
\hline $\begin{array}{l}\text { Groot and colleagues, } \\
\text { 2006 [28] }\end{array}$ & $\begin{array}{l}\text { Combinations of individual stage I to IV } \\
\text { treatment and an extensive mammography } \\
\text { screening control program }\end{array}$ & $\begin{array}{l}\text { To assess the cost-effectiveness of breast } \\
\text { cancer control that covers various } \\
\text { interventions in different settings }\end{array}$ \\
\hline $\begin{array}{l}\text { Okonkwo and } \\
\text { colleagues, 2008 [30] }\end{array}$ & $\begin{array}{l}\text { Mammography screening, CBE screening } \\
\text { among different age groups and in different } \\
\text { frequencies }\end{array}$ & $\begin{array}{l}\text { To assess which screening program } \\
\text { should be implemented in India }\end{array}$ \\
\hline Munshi, 2009 [41] & Several treatment interventions & $\begin{array}{l}\text { To present pragmatic cost-saving breast } \\
\text { cancer interventions }\end{array}$
\end{tabular}

\section{Conclusions by authors}

Stage I treatment and an extensive screening control program are the most cost-effective interventions

CBE screening in India compares favorably with mammography screening in developed countries

Intelligent use of knowledge about
the disease can help us to exploit new techniques for maximum therapeutic gain with minimal investment
Sarvazyan and colleagues, 2008 [32]

$\mathrm{CBE}$, mammography, ultrasound, magnetic resonance imaging, biopsy, elasticity imaging, tactile imaging

\begin{tabular}{ll}
\hline Fonseca and & Anastrozole vs. tamoxifen in the adjuvant \\
colleagues, 2009 [38] & setting of early breast cancer
\end{tabular}
colleagues, 2012 [27]
Ginsberg and
Stage 1 to 4 treatment individual, treatment of all stages, biennial mammography screening 50 to 70 vs. null scenario
To review the diagnostic accuracy, procedure cost, and cost-effectiveness of currently available techniques for breast screening and diagnosis.

To determine cost-effectiveness of anastrozole, compared with tamoxifen, in the adjuvant treatment of early stage breast cancer in Brazil

To determine the cost-effectiveness of 81 interventions to combat breast, cervical and colorectal cancer at different geographic coverage levels, to guide resource allocation decisions in LMICS

\begin{tabular}{ll}
\hline Salomon and & Stage 1 to 4 treatment individual, treatment \\
colleagues, 2012 [31] & of all stages, screening (annual CBE $>25$ \\
& years + mammography annual $>50$ years + \\
& mammography biennial $>40$ to 49 years) \\
& vs. null scenario
\end{tabular}

Analyze the cost-effectiveness of 101 intervention strategies directed at nine major clusters of NCDs in Mexico (including breast cancer), to inform decision-makers

Pakseresht and NA

colleagues, 2011 [48]

A

(n)

To estimate the expenditure audit of
women with breast cancer in a tertiary
hospital in Delhi

\begin{tabular}{ll}
\hline $\begin{array}{l}\text { Szynglarewicz and } \\
\text { Matkowski, } 2011 \text { [33] }\end{array}$ & $\begin{array}{l}\text { Polish screening program costs vs. other } \\
\text { countries }\end{array}$ \\
\hline $\begin{array}{ll}\text { Yazihan and Yilmaz, } & \text { Mammography screening in age group } \\
2006 \text { [34] } & 50 \text { to } 69 \text { vs. treatment only }\end{array}$
\end{tabular}
To show preliminary results of the Polish screening program provide cost-effective breast cancer screening and diagnosis

\section{Anastrozole is more cost-effective} than tamoxifen in the adjuvant setting of early breast cancer mammography screening in combination with treatment of all (I\$2,248 to 4,596/DALY). Treating early-stage breast cancer is more cost-effective than treating late-stage disease

Treatment of all stages is costeffective and treatment of early stages is more cost-effective than late stage treatment. Nationwide screening has an incremental CEA of $\mathbf{\$} 22,000 / D A L Y$ and is potentially cost-effective cancer depends on many factors, including the size and stage of the cancer, the woman's age, use of private hospitals and insurance

Population-based mammographic quality standards is cost-effective even for middle-income countries

\section{To determine the efficiency of resource} usage in mammography screenings

Mammography screening is economically attractive for Turkey
Tactile imaging has the potential to

For breast cancer, although expensive, stages is cost-effective in both regions

Expenditure on treatment for breast screening conforming the European and the impact on breast cancer stages in Turkey

Bastani and Kiadaliri, Docetaxel, doxorubicin and To evaluate the cost-utility of TAC 2012 [49] cyclophosphamide (TAC) vs. 5-fluorouracil, and FAC in node-positive breast doxorubicin, cyclophosphamide (FAC) in cancer patients node-positive breast cancer patients

\begin{tabular}{lll}
\hline Liubao and & AC (doxorubicin/cyclophosphamide) vs. TC & To estimate the cost-effectiveness of \\
colleagues, 2009 [39] & (docetaxel/cyclophosphamide) & AC (doxorubicin/cyclophosphamide) \\
& & vs. TC (docetaxel/cyclophosphamide)
\end{tabular}

Astim, 2011 [36] Annual and biennial mammography screening in various age groups $(40+, 45+, 50+, 55+, 60+$ years $)$ vs. no screening

Zelle and colleagues, Treatment interventions, biennial 2012 [35] mammography and CBE screening interventions, awareness raising
FAC was a dominant option versus TAC in the short term. In this study, TAC resulted in higher costs and lower QALYs over the study period

TC appears to be more effective and more costly than AC. TC may be viewed as cost-effective using the general WHO threshold

Results of the simulation suggests

that women over 40 in Turkey should be screened by mammography biennially

Both screening by clinical breast examination and mass media awareness raising seem 


\section{Table 3 Interventions compared, study objectives, and main study conclusions of reviewed articles (Continued)}

\begin{tabular}{|c|c|c|c|}
\hline & $\begin{array}{l}\text { interventions, palliative care interventions } \\
\text { vs. null scenario }\end{array}$ & $\begin{array}{l}\text { identify the optimal mix of interventions } \\
\text { to maximize population health }\end{array}$ & $\begin{array}{l}\text { economically attractive interventions } \\
(\$ 1,299 \text { and } \$ 1,364 / D A L Y) \text {. } \\
\text { Mammography screening is not } \\
\text { cost-effective }\end{array}$ \\
\hline $\begin{array}{l}\text { Bai and colleagues, } \\
2012 \text { [42] }\end{array}$ & $\begin{array}{l}\text { Radiotherapy vs. no radiotherapy after } \\
\text { surgery }\end{array}$ & $\begin{array}{l}\text { To assess the cost-effectiveness of } \\
\text { additional radiotherapy for women } \\
\text { with early breast cancer after } \\
\text { breast-conserving surgery }\end{array}$ & $\begin{array}{l}\text { In health resource-limited settings, the } \\
\text { addition of radiotherapy is a very } \\
\text { cost-effective strategy (-\$420/ QALY) } \\
\text { in comparison with no-radio therapy } \\
\text { in women with early breast cancer }\end{array}$ \\
\hline $\begin{array}{l}\text { Arredondo and } \\
\text { colleagues, } 1995 \text { [43] }\end{array}$ & $\begin{array}{l}\text { Case management costs for infrastructure, } \\
\text { human resources, laboratory, hospital stay, } \\
\text { drugs, mastectomy, disposable material, } \\
\text { curing material }\end{array}$ & $\begin{array}{l}\text { To develop a system for monitoring } \\
\text { costs of case management for each } \\
\text { disease (breast cancer, cardiac calve } \\
\text { disease and enteritis and } \\
\text { bronchopneumonia) }\end{array}$ & $\begin{array}{l}\text { Economic analyses hold important } \\
\text { information for decision-making }\end{array}$ \\
\hline $\begin{array}{l}\text { Boutayeb and } \\
\text { colleagues, } 2010 \text { [37] }\end{array}$ & $\begin{array}{l}\text { Three chemotherapy regimes, } \mathrm{AC}, \mathrm{AC}+ \\
\text { taxanes, } \mathrm{AC}+\text { taxanes }+ \text { trastuzumab }\end{array}$ & $\begin{array}{l}\text { To evaluate the total cost of } \\
\text { chemotherapy in early stage } \\
\text { breast cancer }\end{array}$ & $\begin{array}{l}\text { Moroccan health authorities need to } \\
\text { devote between US\$13.3 to } 28.6 \\
\text { million to treat women by } \\
\text { chemotherapy every year }\end{array}$ \\
\hline $\begin{array}{l}\text { Denewer and } \\
\text { colleagues, } 2010 \text { [26] }\end{array}$ & $\begin{array}{l}\text { CBE-based screening with selective } \\
\text { mammography vs. no screening }\end{array}$ & $\begin{array}{l}\text { To evaluate the disease pattern of } \\
\text { screen-detected cancers and determine } \\
\text { the effectiveness of CBE-based screening }\end{array}$ & $\begin{array}{l}\text { CBE-based screening with selective } \\
\text { mammography is feasible, effective } \\
\text { and improves the results of breast } \\
\text { cancer management in Egypt }\end{array}$ \\
\hline $\begin{array}{l}\text { Guggisberg and } \\
\text { colleagues, } 2011 \text { [46] }\end{array}$ & On-site FNA clinic vs. shipping of specimens & $\begin{array}{l}\text { To assess the feasibility of an on-site } \\
\text { cytopathology clinic in a rural hospital } \\
\text { in Cameroon }\end{array}$ & $\begin{array}{l}\text { Cytopathology (FNA) is a reliable } \\
\text { alternative for tissue diagnosis in } \\
\text { low-resource settings }\end{array}$ \\
\hline Kobayashi, 1988 [44] & $\begin{array}{l}\text { Costs and performance of breast } \\
\text { echography in different institutions }\end{array}$ & $\begin{array}{l}\text { To analyze the economics and cost } \\
\text { performance of breast echography in } \\
\text { various institutions }\end{array}$ & $\begin{array}{l}\text { The best cost performance, } \\
\text { internationally, can be achieved by } \\
\text { mechanical and real-time electronic } \\
\text { linear scanners }\end{array}$ \\
\hline $\begin{array}{l}\text { Love and colleagues, } \\
2002[40]\end{array}$ & $\begin{array}{l}\text { Adjuvant oophorectomy and tamoxifen } \\
\text { vs. oophorectomy and tamoxifen for } \\
\text { recurrence after observation. }\end{array}$ & $\begin{array}{l}\text { To evaluate costs, disease-free and } \\
\text { overall survival after surgical } \\
\text { oophorectomy and tamoxifen in } \\
\text { premenopausal Vietnamese women } \\
\text { with operable breast cancer }\end{array}$ & $\begin{array}{l}\text { Vietnamese and Chinese women with } \\
\text { hormone receptor-positive operable } \\
\text { breast cancer benefit from adjuvant } \\
\text { treatment with surgical } \\
\text { oophorectomy and tamoxifen }\end{array}$ \\
\hline $\begin{array}{l}\text { Mousavi and } \\
\text { colleagues, } 2008 \text { [29] }\end{array}$ & $\begin{array}{l}\text { Mammography screening in age groups } \\
35 \text { to } 69 \text { and } 50 \text { to } 69 \text { and no screening }\end{array}$ & $\begin{array}{l}\text { To decide whether mammography } \\
\text { screening should be established in Iran } \\
\text { or whether other options are needed }\end{array}$ & $\begin{array}{l}\text { Benefits of other policies than } \\
\text { mammography screening need } \\
\text { to be explored }\end{array}$ \\
\hline $\begin{array}{l}\text { Nasrinossadat and } \\
\text { colleagues, } 2011[47]\end{array}$ & $\begin{array}{l}\text { Methylene blue dye injections vs. wire } \\
\text { localization }\end{array}$ & $\begin{array}{l}\text { To report experience in marking } \\
\text { nonpalpable breast masses by injection } \\
\text { of methylene dye }\end{array}$ & $\begin{array}{l}\text { Marking with methylene blue dye is } \\
\text { a simple, effective and low-cost } \\
\text { method for localization of } \\
\text { nonpalpable breast masses }\end{array}$ \\
\hline $\begin{array}{l}\text { Thomas and } \\
\text { colleagues, } 1999 \text { [45] }\end{array}$ & FNA cytology vs. surgical tissue biopsy & $\begin{array}{l}\text { To assess the results and limitations of a } \\
\text { Nigerian FNA clinic }\end{array}$ & $\begin{array}{l}\text { FNA cytology can help improve the } \\
\text { management and cost of care of } \\
\text { patients with palpable masses }\end{array}$ \\
\hline
\end{tabular}

CEA, cost-effectiveness analysis; CBE, clinical breast examination; DALY, disability-adjusted life year; FNA, fine needle aspiration; LMIC, low- and middle-income country; NCD, noncommunicable disease; QALY, quality-adjusted life year; WHO, World Health Organization.

\section{Study quality}

Table 4 summarizes the quality of the included studies, as indicated by the percentage score. The quality of all studies ranges from 23 to $86 \%$. Studies by Ginsberg and colleagues, Zelle and colleagues, and Bai and colleagues had the highest total average scores, and these were all modeling studies $[27,35,42]$. If items were not applicable (NA) for a reviewed paper, the maximum obtainable (domain) score was reduced with 2 points per item.

Studies generally scored poorly on the domain 'estimation of costs', at an average $34 \%$ of the maximum obtainable score across all studies. The average score for 'study design' was $73 \%$, while the quality of the domains 'estimation of effectiveness,' 'analysis', and 'interpretation of results' was scores as $70 \%, 51 \%$, and $68 \%$, respectively.

\section{Study findings}

As described earlier, most studies evaluated breast cancer screening in combination with treatment. Studies in Mexico, Poland, Turkey identified mammography screening as a cost-effective intervention [31,33,34,36], whereas studies in India, Ghana and Egypt found other strategies (such as CBE screening or mass-media awareness raising) to be economically more attractive (Table 3) [26,30,35]. Sarvazyan and colleagues proposed another breast cancer 
Table 4 Summary of quality assessment and domain scores of reviewed studies

\begin{tabular}{|c|c|c|c|c|c|c|c|c|c|}
\hline \multirow[t]{2}{*}{ Authors } & & \multirow[b]{2}{*}{$\begin{array}{l}\text { Study } \\
\text { design }\end{array}$} & \multicolumn{4}{|c|}{ Scored domains } & \multicolumn{3}{|c|}{ Summary scores } \\
\hline & & & $\begin{array}{l}\text { Effectiveness } \\
\text { estimation }\end{array}$ & $\begin{array}{l}\text { Cost } \\
\text { estimation }\end{array}$ & Analysis & $\begin{array}{l}\text { Interpretation } \\
\text { of results }\end{array}$ & $\begin{array}{l}\text { Number of } \\
\text { items scored }\end{array}$ & $\begin{array}{l}\text { Sum of } \\
\text { scores }\end{array}$ & $\begin{array}{l}\text { Total } \\
\text { averag } \\
\text { score }\end{array}$ \\
\hline \multirow{2}{*}{$\begin{array}{l}\text { Groot and colleagues, } \\
2006 \text { [28] }\end{array}$} & Score granted & 12 & 7 & 6 & 16 & 9 & 29 & 50 & 1.72 \\
\hline & $\begin{array}{l}\% \text { of maximum } \\
\text { (domain) score }\end{array}$ & $86 \%$ & $88 \%$ & $75 \%$ & $89 \%$ & $90 \%$ & & & $86 \%$ \\
\hline \multirow{2}{*}{$\begin{array}{l}\text { Okonkwo and } \\
\text { colleagues, } 2008 \text { [30] }\end{array}$} & Score granted & 12 & 6 & 3 & 16 & 10 & 28 & 47 & 1.68 \\
\hline & $\begin{array}{l}\% \text { of maximum } \\
\text { (domain) score }\end{array}$ & $86 \%$ & $100 \%$ & $38 \%$ & $100 \%$ & $100 \%$ & & & $84 \%$ \\
\hline \multirow[t]{2}{*}{ Munshi, 2009 [41] } & Score granted & 7 & 7 & 0 & 1 & 4 & 21 & 19 & 0.90 \\
\hline & $\begin{array}{l}\% \text { of maximum } \\
\text { (domain) score }\end{array}$ & $50 \%$ & $70 \%$ & $0 \%$ & $50 \%$ & $40 \%$ & & & $45 \%$ \\
\hline \multirow{2}{*}{$\begin{array}{l}\text { Sarvazyan and } \\
\text { colleagues, } 2008 \text { [32] }\end{array}$} & Score granted & 7 & 7 & 0 & 1 & 4 & 21 & 19 & 0.90 \\
\hline & $\begin{array}{l}\% \text { of maximum } \\
\text { (domain) score }\end{array}$ & $50 \%$ & $70 \%$ & $0 \%$ & $50 \%$ & $40 \%$ & & & $45 \%$ \\
\hline \multirow{2}{*}{$\begin{array}{l}\text { Fonseca and } \\
\text { colleagues, } 2009 \text { [38] }\end{array}$} & Score granted & 14 & 6 & 1 & 13 & 10 & 28 & 44 & 1.57 \\
\hline & $\begin{array}{l}\% \text { of maximum } \\
\text { (domain) score }\end{array}$ & $100 \%$ & $100 \%$ & $13 \%$ & $72 \%$ & $100 \%$ & & & $79 \%$ \\
\hline \multirow{2}{*}{$\begin{array}{l}\text { Ginsberg and } \\
\text { colleagues, } 2012 \text { [27] }\end{array}$} & Score granted & 12 & 8 & 8 & 18 & 10 & 29 & 52 & 1.79 \\
\hline & $\begin{array}{l}\% \text { of maximum } \\
\text { (domain) score }\end{array}$ & $86 \%$ & $100 \%$ & $75 \%$ & $89 \%$ & $100 \%$ & & & $90 \%$ \\
\hline \multirow{2}{*}{$\begin{array}{l}\text { Salomon and } \\
\text { colleagues, } 2012 \text { [31] }\end{array}$} & Score granted & 12 & 6 & 5 & 14 & 8 & 29 & 45 & 1.55 \\
\hline & $\begin{array}{l}\text { \% of maximum } \\
\text { (domain) score }\end{array}$ & $86 \%$ & $75 \%$ & $63 \%$ & $78 \%$ & $80 \%$ & & & $78 \%$ \\
\hline \multirow{2}{*}{$\begin{array}{l}\text { Pakseresht and } \\
\text { colleagues, } 2011 \text { [48] }\end{array}$} & Score granted & 7 & 1 & 4 & 3 & 5 & 15 & 20 & 1.33 \\
\hline & $\begin{array}{l}\% \text { of maximum } \\
\text { (domain) score }\end{array}$ & $88 \%$ & $50 \%$ & $50 \%$ & $75 \%$ & $63 \%$ & & & $67 \%$ \\
\hline \multirow{2}{*}{$\begin{array}{l}\text { Szynglarewicz and } \\
\text { Matkowski, } 2011 \text { [33] }\end{array}$} & Score granted & 5 & 3 & 2 & 1 & 5 & 24 & 15 & 0.625 \\
\hline & $\begin{array}{l}\text { \% of maximum } \\
\text { (domain) score }\end{array}$ & $88 \%$ & $50 \%$ & $50 \%$ & $75 \%$ & $63 \%$ & & & $33 \%$ \\
\hline \multirow{2}{*}{$\begin{array}{l}\text { Yazihan and Yilmaz, } \\
2006 \text { [34] }\end{array}$} & Score granted & 12 & 0 & 3 & 2 & 5 & 28 & 22 & 0.79 \\
\hline & $\begin{array}{l}\% \text { of maximum } \\
\text { (domain) score }\end{array}$ & $86 \%$ & $0 \%$ & $38 \%$ & $13 \%$ & $50 \%$ & & & $40 \%$ \\
\hline \multirow{2}{*}{$\begin{array}{l}\text { Bastani and Kiadaliri, } \\
2012 \text { [49] }\end{array}$} & Score granted & 13 & 8 & 4 & 7 & 8 & 25 & 40 & 1.6 \\
\hline & $\begin{array}{l}\% \text { of maximum } \\
\text { (domain) score }\end{array}$ & $93 \%$ & $100 \%$ & $50 \%$ & $70 \%$ & $80 \%$ & & & $80 \%$ \\
\hline \multirow{2}{*}{$\begin{array}{l}\text { Liubao and } \\
\text { colleagues, } 2009 \text { [39] }\end{array}$} & $\underline{\text { Score granted }}$ & 13 & 7 & 4 & 16 & 10 & 29 & 50 & 1.72 \\
\hline & $\begin{array}{l}\% \text { of maximum } \\
\text { (domain) score }\end{array}$ & $93 \%$ & $88 \%$ & $50 \%$ & $89 \%$ & $100 \%$ & & & $86 \%$ \\
\hline \multirow[t]{2}{*}{ Astim, 2011 [36] } & Score granted & 9 & 5 & 3 & 8 & 7 & 28 & 32 & 1.14 \\
\hline & $\begin{array}{l}\% \text { of maximum } \\
\text { (domain) score }\end{array}$ & $64 \%$ & $63 \%$ & $38 \%$ & $50 \%$ & $70 \%$ & & & $57 \%$ \\
\hline \multirow{2}{*}{$\begin{array}{l}\text { Zelle and colleagues, } \\
2012 \text { [35] }\end{array}$} & $\underline{\text { Score granted }}$ & 14 & 7 & 7 & 14 & 10 & 29 & 52 & 1.79 \\
\hline & $\begin{array}{l}\% \text { of maximum } \\
\text { (domain) score }\end{array}$ & $100 \%$ & $88 \%$ & $88 \%$ & $78 \%$ & $100 \%$ & & & $90 \%$ \\
\hline \multirow{2}{*}{$\begin{array}{l}\text { Bai and colleagues, } \\
2012 \text { [42] }\end{array}$} & Score granted & 13 & 8 & 5 & 18 & 8 & 29 & 52 & 1.79 \\
\hline & $\begin{array}{l}\% \text { of maximum } \\
\text { (domain) score }\end{array}$ & $93 \%$ & $100 \%$ & $63 \%$ & $100 \%$ & $80 \%$ & & & $90 \%$ \\
\hline \multirow{3}{*}{$\begin{array}{l}\text { Arredondo and } \\
\text { colleagues, } 1995 \text { [43] }\end{array}$} & Score granted & 10 & NA & 1 & 0 & 7 & 18 & 18 & 1.00 \\
\hline & $\begin{array}{l}\% \text { of maximum } \\
\text { (domain) score }\end{array}$ & $71 \%$ & NA & $13 \%$ & $0 \%$ & $70 \%$ & & & $50 \%$ \\
\hline & Score granted & 12 & 4 & 4 & 1 & 6 & 25 & 27 & 1.08 \\
\hline
\end{tabular}


Table 4 Summary of quality assessment and domain scores of reviewed studies (Continued)

\begin{tabular}{|c|c|c|c|c|c|c|c|c|c|}
\hline $\begin{array}{l}\text { Boutayeb and } \\
\text { colleagues, } 2010 \text { [37] }\end{array}$ & $\begin{array}{l}\text { \% of maximum } \\
\text { (domain) score }\end{array}$ & $86 \%$ & $50 \%$ & $50 \%$ & $13 \%$ & $60 \%$ & & & $54 \%$ \\
\hline \multirow{2}{*}{$\begin{array}{l}\text { Denewer and } \\
\text { colleagues, } 2010 \text { [26] }\end{array}$} & Score granted & 10 & 4 & 0 & 2 & 5 & 25 & 21 & 0.84 \\
\hline & $\begin{array}{l}\text { \% of maximum } \\
\text { (domain) score }\end{array}$ & $71 \%$ & $50 \%$ & $0 \%$ & $20 \%$ & $50 \%$ & & & $42 \%$ \\
\hline \multirow{2}{*}{$\begin{array}{l}\text { Guggisberg and } \\
\text { colleagues, } 2011 \text { [46] }\end{array}$} & Score granted & 3 & 6 & 2 & 1 & 5 & 25 & 24 & 0.96 \\
\hline & $\begin{array}{l}\text { \% of maximum } \\
\text { (domain) score }\end{array}$ & $21 \%$ & $75 \%$ & $25 \%$ & $13 \%$ & $50 \%$ & & & $35 \%$ \\
\hline \multirow[t]{2}{*}{ Kobayashi, 1988 [44] } & Score granted & 4 & 4 & 1 & NA & 3 & 19 & 12 & 0.63 \\
\hline & $\begin{array}{l}\% \text { of maximum } \\
\text { (domain) score }\end{array}$ & $29 \%$ & $67 \%$ & $13 \%$ & NA & $30 \%$ & & & $32 \%$ \\
\hline \multirow{2}{*}{$\begin{array}{l}\text { Love and colleagues, } \\
2002 \text { [40] }\end{array}$} & Score granted & 9 & 6 & 1 & 10 & 8 & 27 & 34 & 1.26 \\
\hline & $\begin{array}{l}\text { \% of maximum } \\
\text { (domain) score }\end{array}$ & $64 \%$ & $100 \%$ & $13 \%$ & $63 \%$ & $80 \%$ & & & $63 \%$ \\
\hline \multirow{2}{*}{$\begin{array}{l}\text { Mousavi and } \\
\text { colleagues, } 2008 \text { [29] }\end{array}$} & Score granted & 5 & 1 & 0 & 1 & 3 & 22 & 10 & 0.45 \\
\hline & $\begin{array}{l}\text { \% of maximum } \\
\text { (domain) score }\end{array}$ & $36 \%$ & $25 \%$ & $0 \%$ & $13 \%$ & $30 \%$ & & & $23 \%$ \\
\hline \multirow{2}{*}{$\begin{array}{l}\text { Nasrinossadat and } \\
\text { colleagues, } 2011 \text { [47] }\end{array}$} & Score granted & 75 & 5 & 0 & 0 & 5 & 25 & 17 & 0.68 \\
\hline & $\begin{array}{l}\text { \% of maximum } \\
\text { (domain) score }\end{array}$ & $50 \%$ & $63 \%$ & $0 \%$ & $0 \%$ & $50 \%$ & & & $34 \%$ \\
\hline \multirow{2}{*}{$\begin{array}{l}\text { Thomas and } \\
\text { colleagues, } 1999 \text { [45] }\end{array}$} & Score granted & 7 & 4 & 0 & 0 & 6 & 21 & 17 & 0.81 \\
\hline & $\begin{array}{l}\text { \% of maximum } \\
\text { (domain) score }\end{array}$ & $50 \%$ & $67 \%$ & $0 \%$ & $0 \%$ & $60 \%$ & & & $41 \%$ \\
\hline \multicolumn{2}{|c|}{ Total average domain score (\%) } & $73 \%$ & $70 \%$ & $34 \%$ & $51 \%$ & $68 \%$ & & & \\
\hline
\end{tabular}

screening option: tactile imaging as an alternative to several other interventions [32].

Studies evaluating treatment interventions typically favored the novel interventions. Anastrozole was more cost-effective than tamoxifen in a Brazilian study [38], oophorectomy and tamoxifen after recurrence was shown to be favorable in Vietnamese and Chinese patients [40], additional radiotherapy after breast-conserving surgery was very cost-effective in China [42], and chemotherapy consisting of a docetaxel and cyclophosphamide regimen was more attractive compared with an doxorubicin and cyclophosphamide regimen also in Chinese patients [39]. There was only one study with a negative suggestion for the novel and more costly intervention docetaxel, doxorubicin, cyclophosphamide, as compared with the more conventional 5-fluorouracil, doxorubicin, dyclophosphamide regime [49].

Studies that only assessed costs and did not include effectiveness estimates, reported on costs of breast cancer for patient management in Brazil (US\$1,646 per patient) [43], and the costs of patient expenditure (US\$242 per patient) in India [48].

The three studies evaluating diagnostic interventions demonstrated the economic attractiveness of inexpensive interventions; that is, fine-needle aspiration cytology and methylene blue dye injections [45-47]. These interventions could be especially relevant for diagnosing breast cancer in rural settings and settings with low resources.

\section{Discussion}

This study shows that there is limited economic evidence on breast cancer control in LMICs. Only 24 economic evaluation studies were found in this review, and their quality was generally poor. Furthermore, the study populations were very diverse, as most studies examined different kinds of screening and therapeutic interventions in various age and risk groups. Owing to this poor availability, quality, and comparability, we conclude that the economic evidence base to guide strategies for breast cancer in LMICs is currently insufficient.

Our review raises a few discussion points. First, there is mixed evidence on the economic attractiveness of mammography screening. Studies in Mexico, Poland and Turkey demonstrate the intervention to be cost-effective, whereas studies in India, Ghana, and Egypt suggests that other forms of screening - for example, by CBE provide more value for money. The evidence base is too small to generalize these findings to other LMICs, and to draw general conclusions. Also, most of the studies evaluating therapeutic interventions seem to favor the more novel - and often more expensive - therapy. These findings may be explained by many reasons, including the higher effectiveness of the novel interventions but possibly also the association between funding sources and pro-industry conclusions [50].

Second, in general, we found that the quality of the reviewed articles was poor. The majority of studies failed 
to score at least $50 \%$ on every domain ('study design', 'estimation of effectiveness', 'estimation of costs,' 'analysis', and 'interpretation of results'). These domain scores further show that most emphasis was given to the design of the studies and the interpretation of results, whereas costs, in particular, were poorly evaluated. This calls for better adherence of studies to methodological standards for economic analyses, or the development of such standards specifically for breast cancer research. Future studies could be improved by using a checklist, and through transparent reporting of the items in checklists [25,51].

Third, the current evidence base leaves many LMICs with the difficult task of extrapolating results from other countries. The transferability of economic evaluations across countries is complicated, as clinical practice patterns, healthcare systems, and cultural and ethical practices differ across countries [52,53]. Standardized ways of adopting economic evaluations, with the help of available checklists and guidelines [24,25,51,54-58], may improve this lack of transferability. Alternatively, modeling studies could play an important role in extrapolating results from one context to another. Modeling studies, however, rely on the availability of costing and effectiveness data, and this emphasizes the need for more primary data collection on these aspects in LMICs. With data from such studies, researchers would not have to continue to rely on sensitivity analyses or extrapolating cost estimates from data in HICs. National cancer registries, mortality databases, hospital registries, and accessible publications would be essential for providing such information [59].

Fourth, and closely related, we generally advocate the use of modeling studies in the economic analysis of breast cancer control in LMICs. In addition to their use in the extrapolation of study findings, they generally appeared to be of high quality, are sufficiently flexible to include important methodological characteristics such as adequate time horizon, and seem also appropriate to evaluate a broad array of interventions across different groups.

Fifth, the most adopted type of economic evaluation was cost-effectiveness analysis, using a healthcare perspective and life years saved as the primary outcome. Although cost-effectiveness analyses using a healthcare perspective contribute very important information, productivity losses for patients suffering from breast cancer - and most probably other NCDs - can be substantial $[60,61]$. So far, there is no methodological consensus on estimating productivity loss and the cost of illness can vary greatly between different costing approaches (for example, human capital approach vs. friction cost approach) and also between gender, age and the type of job of patients [62]. Further research should account for economic and social characteristics of the population under study, and should try to investigate productivity losses. Additionally, life years saved may be a less appropriate outcome when palliative or preventive interventions are investigated, and the use of disability-adjusted or quality-adjusted life years may be more appropriate.

Sixth, there is currently very little economic evidence on less established interventions such as tactile imaging, awareness raising, $\mathrm{CBE}$ screening, or preventive and palliative interventions. Economic studies, especially in LMICs, should aim to evaluate these interventions more often (and thereby including broad target populations) as they have the potential to be economically attractive $[26,30,32,35]$.

Finally, guidance in decision-making and recommendations for implementation are generally underemphasized in economic evaluations. By reflecting on the health system characteristics of the particular country and considering them in implementation recommendations, economic evaluations could improve their use in breast cancer policy development.

Our study has a number of limitations. Primarily, the number of articles reviewed is very limited, possibly the result of our search strategy. Besides a possible publication bias - studies with negative outcomes are less likely to be published - we searched only for articles published in English. This may explain the relatively small number of articles found, for instance, from Spanish-speaking regions or from countries where there is less emphasis on publishing research (for example, in Africa). Also, the studies included in our review vastly differed with regard to their methodology, objectives, characteristics, and study populations and hence are difficult to compare. In addition, our quality assessment of the reviewed articles was based on a checklist that gives highest scores to a full reporting of all domains. However, short reports in the form of, for example, editorials may not include all these details but may nevertheless be valid for the goals they serve. Hence, the scores for these studies should be interpreted with caution.

\section{Conclusions}

To conclude, our findings indicate that research on the costs and cost-effectiveness of breast cancer control in LMICs is still in its infancy. The limited evidence base suggests that screening strategies may be economically attractive in LMICs - yet there is very little evidence to provide specific recommendations (on screening by mammography vs. CBE, the frequency of screening, or the target population). These results demonstrate the need for more economic analysis that are uniform, of better quality, cover a comprehensive set of interventions and result in clear policy recommendations.

\section{Abbreviations}

CBE: Clinical breast examination; HIC: High-income country; LMIC: Low- and middle-income country; NCD: Noncommunicable disease. 


\section{Competing interests}

The authors declare that they have no competing interests.

\section{Authors' contributions}

SGZ performed the search strategy, designed the inclusion criteria, reviewed all papers included in the review, developed the evaluation strategy and drafted the manuscript. RMB participated in the design of the study, the selection of relevant articles, the evaluation and classification of articles and contributed to the writing of the manuscript. Both authors reviewed and critically assessed the papers included in this review.

\section{Acknowledgements}

The authors thank Dr Ernst Spaan and Evelinn Mikkelsen for sharing their experience and search strategy on a large health insurance review, which has helped us in setting up our search strategy and with maintaining our search results.

\section{Financial disclosure}

No direct funding was received for this study. SGZ and RMB were salaried by their institutions during the period of writing though no specific salary was set aside or given for the writing of this paper. No funding bodies had any role in the study design, data collection, analysis, decision to publish or preparation of the manuscript.

Received: 16 November 2012 Accepted: 4 March 2013

Published: 8 April 2013

\section{References}

1. Abegunde DO, Mathers CD, Adam T, Ortegon M, Strong K: The burden and costs of chronic diseases in low-income and middle-income countries. Lancet 2007, 370:1929-1938.

2. Beaglehole R, Bonita R, Horton R, Adams C, Alleyne G, Asaria P, Baugh V, Bekedam H, Billo N, Casswell S, Cecchini M, Colagiuri R, Colagiuri S, Collins T, Ebrahim S, Engelgau M, Galea G, Gaziano T, Geneau R, Haines A, Hospedales J, Jha P, Keeling A, Leeder S, Lincoln P, McKee M, Mackay J, Magnusson R, Moodie R, Mwatsama M, Lancet NCDAG, Alliance NCD: Priority actions for the non-communicable disease crisis. Lancet 2011, 377:1438-1447.

3. Ferlay J, Shin HR, Bray F, Forman D, Mathers C, Parkin DM: Estimates of worldwide burden of cancer in 2008: GLOBOCAN. Int J Cancer 2008, 127:2893-2917.

4. The good news about cancer in developing countries. Lancet 2011, 378:1605.

5. Jemal A, Thun MJ, Ries LA, Howe HL, Weir HK, Center MM, Ward E, Wu XC, Eheman C, Anderson R, Ajani UA, Kohler B, Edwards BK: Annual report to the nation on the status of cancer, 1975-2005, featuring trends in lung cancer, tobacco use, and tobacco control. J Natl Cancer Inst 2008, 100:1672-1694.

6. Levi F, Lucchini F, Negri E, La Vecchia C: Continuing declines in cancer mortality in the European Union. Ann Oncol 2007, 18:593-595.

7. Duffy SW, Tabar L, Olsen AH, Vitak B, Allgood PC, Chen TH, Yen AM, Smith RA: Absolute numbers of lives saved and overdiagnosis in breast cancer screening, from a randomized trial and from the Breast Screening Programme in England. J Med Screen 2010, 17:25-30.

8. Gotzsche PC, Hartling OJ, Nielsen M, Brodersen J, Jorgensen KJ: Breast screening: the facts - or maybe not. BMJ 2009, 338:b86

9. Kopans DB, Smith RA, Duffy SW: Mammographic screening and 'overdiagnosis'. Radiology 2011, 260:616-620.

10. Boyle P, Levin B: World Cancer Report 2008. Lyon: International Agency for Research on Cancer (IARC); 2008.

11. Coleman MP, Forman D, Bryant H, Butler J, Rachet B, Maringe C, Nur U, Tracey E, Coory M, Hatcher J, McGahan CE, Turner D, Marrett L, Gjerstorff ML, Johannesen TB, Adolfsson J, Lambe M, Lawrence G, Meechan D, Morris EJ, Middleton R, Steward J, Richards MA: Cancer survival in Australia, Canada, Denmark, Norway, Sweden, and the UK, 1995-2007 (the International Cancer Benchmarking Partnership): an analysis of population-based cancer registry data. Lancet 2011, 377:127-138.

12. Vainio $\mathrm{H}$, Bianchini F: IARC Handbook of Cancer Prevention. Breast Cancer Screening. 7th edition. Lyon: IARC Press; 2002.

13. De Koning $\mathrm{HJ}$ : Breast cancer screening; cost-effective in practice? Eur J Radiol 2000, 33:32-37.

14. Eddy DM: Screening for breast cancer. Ann Intern Med 1989, 111:389-399.
15. Lindfors KK, Rosenquist CJ: The cost-effectiveness of mammographic screening strategies. JAMA 1995, 274:881-884.

16. Stout NK, Rosenberg MA, Trentham-Dietz A, Smith MA, Robinson SM, Fryback DG: Retrospective cost-effectiveness analysis of screening mammography. J Natl Cancer Inst 2006, 98:774-782.

17. Anderson BO, Cazap E, El Saghir NS, Yip CH, Khaled HM, Otero IV, Adebamowo CA, Badwe RA, Harford JB: Optimisation of breast cancer management in low-resource and middle-resource countries: executive summary of the Breast Health Global Initiative consensus, 2010. Lancet Oncol 2011, 12:387-398.

18. Corbex M, Burton R, Sancho-Garnier H: Breast cancer early detection methods for low and middle income countries, a review of the evidence. Breast (Edinburgh, Scotland) 2012, 21:428-434.

19. Ginsburg OM, Love RR: Breast cancer: a neglected disease for the majority of affected women worldwide. Breast J 2011, 17:289-295.

20. Harford JB, Otero IV, Anderson BO, Cazap E, Gradishar WJ, Gralow JR, Kane GM, Niens LM, Porter PL, Reeler AV, Rieger PT, Shockney LD, Shulman LN, Soldak T, Thomas DB, Thompson B, Winchester DP, Zelle SG, Badwe RA: Problem solving for breast health care delivery in low and middle resource countries (LMCs): consensus statement from the Breast Health Global Initiative. Breast (Edinburgh, Scotland) 2011, 20(Suppl 2):S20-S29.

21. Autier P, Boniol M, La Vecchia C, Vatten L, Gavin A, Hery C, Heanue M: Disparities in breast cancer mortality trends between 30 European countries: retrospective trend analysis of WHO mortality database. BMJ 2010, 341:c3620.

22. World Bank Country Classification. [http://data.worldbank.org/about/ country-classifications].

23. Greene FL, Compton CC, Fritz AG: AJCC Cancer Staging Atlas. New York: Springer; 2006

24. Drummond MF, Jefferson TO: Guidelines for authors and peer reviewers of economic submissions to the BMJ. The BMJ Economic Evaluation Working Party. BMJ (Clin Res Ed) 1996, 313:275-283.

25. Gerard K, Seymour J, Smoker I: A tool to improve quality of reporting published economic analyses. Int J Technol Assess Health Care 2000, 16:100-110.

26. Denewer A, Hussein O, Farouk O, Elnahas W, Khater A, El-Saed A: Cost-effectiveness of clinical breast assessment-based screening in rural Egypt. World J Surg 2010, 34:2204-2210.

27. Ginsberg GM, Lauer JA, Zelle S, Baeten S, Baltussen R: Cost effectiveness of strategies to combat breast, cervical, and colorectal cancer in subSaharan Africa and South East Asia: mathematical modelling study. BMJ 2012, 344:e614.

28. Groot MT, Baltussen R, Uyl-De Groot CA, Anderson BO, HortobÃ igyi GN: Costs and health effects of breast cancer interventions in epidemiologically different regions of Africa, North America, and Asia. Breast J 2006, 12:S81-S90.

29. Mousavi SM, Harirchi I, Ebrahimi M, Mohagheghi MA, Montazeri A, Jarrahi AM, Gouya MM, Miller AB: Screening for breast cancer in Iran: a challenge for health policy makers. Breast J 2008, 14:605-606.

30. Okonkwo QL, Draisma G, der Kinderen A, Brown ML, de Koning HJ: Breast cancer screening policies in developing countries: a cost-effectiveness analysis for India. J Natl Cancer Inst 2008, 100:1290-1300.

31. Salomon JA, Carvalho N, Gutierrez-Delgado C, Orozco R, Mancuso A, Hogan DR, Lee D, Murakami Y, Sridharan L, Medina-Mora ME, Gonzalez-Pier E: Intervention strategies to reduce the burden of non-communicable diseases in Mexico: cost effectiveness analysis. Br Med J 2012, 344:S20-S29.

32. Sarvazyan A, Egorov V, Son JS, Kaufman CS: Cost-effective screening for breast cancer worldwide: current state and future directions. Breast Cancer 2008, 1:91-99.

33. Szynglarewicz B, Matkowski R: Low cost of cancer detection in the first year of mammographic screening in Poland. Breast (Edinburgh, Scotland) 2011, 20:585.

34. Yazihan N, Yilmaz HH: Breast cancer in Turkey: economic efficiency and cost effectiveness. 2006, 740:S20-S29.

35. Zelle SG, Nyarko KM, Bosu WK, Aikins M, Niens LM, Lauer JA, Sepulveda CR Hontelez JA, Baltussen R: Costs, effects and cost-effectiveness of breast cancer control in Ghana. Trop Med Int Health 2012, 17:1031-1043.

36. Astim E: Cost-effectiveness analysis of a prospective breast cancer screening program in Turkey. Thesis. Graduate School of Social Sciences of Middle East Technical University; 2011 [https://etd.lib.metu.edu.tr/upload/12612806/index.pdf].

37. Boutayeb S, Boutayeb A, Ahbeddou N, Boutayeb W, Essaadi I, Tazi M, Errihani H: Estimation of the cost of treatment by chemotherapy for early breast cancer in Morocco. Cost Eff Resour Alloc 2010, 8:16. 
38. Fonseca M, Araujo GT, Saad ED: Cost-effectiveness of anastrozole, in comparison with tamoxifen, in the adjuvant treatment of early breast cancer in Brazil. Rev Assoc Med Bras 2009, 55:410-415.

39. Liubao P, Xiaomin W, Chongqing T, Karnon J, Gannong C, Jianhe L, Wei C, Xia L, Junhua C: Cost-effectiveness analysis of adjuvant therapy for operable breast cancer from a Chinese perspective: doxorubicin plus cyclophosphamide versus docetaxel plus cyclophosphamide. PharmacoEconomics 2009, 27:873-886.

40. Love RR, Duc NB, Allred DC, Binh NC, Dinh NV, Kha NN, Thuan TV, Mohsin SK, Roanh LD, Khang HX, Tran TL, Quy TT, Thuy NV, Thé PN, Cau TT, Tung ND, Huong DT, Quang LM, Hien NN, Thuong L, Shen TZ, Xin Y, Zhang Q, Havighurst TC, Yang YF, Hillner BE, DeMets DL: Oophorectomy and tamoxifen adjuvant therapy in premenopausal Vietnamese and Chinese women with operable breast cancer. J Clin Oncol 2002, 20:2559-2566.

41. Munshi A: Resource-sparing and cost-effective strategies in current management of breast cancer. J Canc Res Ther 2009, 5:116-120.

42. Bai $Y, Y e ~ M, C a o ~ H, M a ~ X, X u ~ Y, W u$ B: Economic evaluation of radiotherapy for early breast cancer after breast-conserving surgery in a health resource-limited setting. Breast Cancer Res $\operatorname{Tr} 2012,136: 547-557$.

43. Arredondo A, Lockett LY, de Icaza E: Cost of diseases in Brazil: breast cancer, enteritis, cardiac valve disease and bronchopneumonia. Rev Saude Publica 1995, 29:349-354.

44. Kobayashi T: International trends in the economics of breast examination. Ultrasound Med Biol 1988, 14(Suppl 1):217-229.

45. Thomas JO, Amanguno AU, Adeyi OA, Adesina AO: Fine needle aspiration (FNA) in the management of palpable masses in Ibadan: impact on the cost of care. Cytopathology 1999, 10:206-210.

46. Guggisberg K, Okorie C, Khalil M: Cytopathology including fine-needle aspiration in sub-Saharan Africa: a Cameroon experience. Arch Pathol Lab Med 2011, 135:200-206.

47. Nasrinossadat A, Ladan F, Fereshte E, Asieh O, Reza C, Akramossadat S, Golshan M: Marking non-palpable breast masses with injected methylene blue dye, an easy, safe and low cost method for developing countries and resource-limited areas. Asian Pac J Cancer P 2011, 12:1189-1192.

48. Pakseresht S, Ingle GK, Garg S, Singh MM: Expenditure audit of women with breast cancer in a tertiary care hospital of Delhi. Indian J Cancer 2011, 48:428-437.

49. Bastani P, Kiadaliri AA: Cost-utility analysis of adjuvant therapies for breast cancer in Iran. Int J Technol Assess 2012, 28:110-114.

50. Gluud LL: Bias in clinical intervention research. Am J Epidemiol 2006, 163:493-501.

51. Gerkens S, Crott R, Cleemput I, Thissen JP, Closon MC, Horsmans Y, Beguin $C$ : Comparison of three instruments assessing the quality of economic evaluations: a practical exercise on economic evaluations of the surgical treatment of obesity. Int J Technol Assess 2008, 24:318-325.

52. Sculpher MJ, Pang FS, Manca A, Drummond MF, Golder S, Urdahl H, Davies LM, Eastwood A: Generalisability in economic evaluation studies in healthcare: a review and case studies. Health Technol Assess 2004, 8:1-192.

53. Sun X, Wang L, Li Y: Methodological issues in cost-effectiveness studies: a brief overview. J Evid Based Med 2010, 3:201-204.

54. Briggs AH, Claxton K, Sculpher MJ: Decision Modelling for Health Economic Evaluation. New York: Oxford University Press; 2006

55. Brazier J, Ratcliffe J, Solomon JA, Tsuchia A: Measuring and Valuing Health Benefits for Economic Evaluation. New York: Oxford University Press; 2007.

56. Drummond MF, Sculpher MJ, Torrance GW, O'Brien BJ, Stoddart GL: Methods for the Economic Evaluation of Health Care Programmes. 3rd edition. New York: Oxford University Press; 2005.

57. Ramsey S, Willke R, Briggs A, Brown R, Buxton M, Chawla A, Cook J, Glick H, Liljas B, Petitti D, Reed S: Good research practices for cost-effectiveness analysis alongside clinical trials: the ISPOR RCT-CEA Task Force report. Value Health 2005, 8:521-533.

58. Tan-Torres Edejer T, Baltussen R, Adam T, Hutubessy R, Acharya A, Evans DB, Murray CJL: Making Choices in Health: WHO Guide to Cost-effectiveness Analysis. Geneva: World Health Organization; 2003.

59. Lodge $M$, Corbex M: Establishing an evidence-base for breast cancer control in developing countries. Breast (Edinburgh, Scotland) 2011, 20(Suppl 2):S65-S69.

60. Bloom DE, Cafiero ET, Jané-Llopis E, Abrahams-Gessel S, Bloom LR, Fathima S: The Global Economic Burden of Non-communicable Diseases. Geneva: World Economic Forum; 2011 [www.weforum.org/EconomicsOfNCD].
61. Broekx S, Hond ED, Torfs R, Remacle A, Mertens R, D'Hooghe T, Neven P, Christiaens MR, Simoens S: The costs of breast cancer prior to and following diagnosis. Eur J Health Econ 2011, 12:311-317.

62. Hanly P, Timmons A, Walsh PM, Sharp L: Breast and prostate cancer productivity costs: a comparison of the human capital approach and the friction cost approach. Value Health 2012, 15:429-436.

doi:10.1186/2046-4053-2-20

Cite this article as: Zelle and Baltussen: Economic analyses of breast cancer control in low- and middle-income countries: a systematic review. Systematic Reviews 2013 2:20.

\section{Submit your next manuscript to BioMed Central and take full advantage of:}

- Convenient online submission

- Thorough peer review

- No space constraints or color figure charges

- Immediate publication on acceptance

- Inclusion in PubMed, CAS, Scopus and Google Scholar

- Research which is freely available for redistribution

Submit your manuscript at www.biomedcentral.com/submit
C) Biomed Central 